ANNALES

POLONICI MATHEMATICI

$87(2005)$

\title{
On families of trajectories of an analytic gradient vector field
}

by AdAm Dzedzej and ZBigniew Szafraniec (Gdańsk)

To the memory of Professor Stanisław Eojasiewicz

\begin{abstract}
For an analytic function $f: \mathbb{R}^{n}, 0 \rightarrow \mathbb{R}, 0$ having a critical point at the origin, we describe the topological properties of the partition of the family of trajectories of the gradient equation $\dot{x}=\nabla f(x)$ attracted by the origin, given by characteristic exponents and asymptotic critical values.
\end{abstract}

1. Introduction. Let $f: \mathbb{R}^{n}, 0 \rightarrow \mathbb{R}, 0$ be an analytic function defined in a neighbourhood of the origin, having a critical point at 0 . We consider the trajectories of the gradient vector field $\dot{x}=\nabla f(x)$. Take $y>0$ such that $-y$ is a regular value of $f$. One can show that there exists a closed set $\Gamma \subset f^{-1}(-y)$ such that a non-trivial trajectory of the gradient field is attracted by the origin if and only if it intersects $f^{-1}(-y)$ transversally at a point belonging to $\Gamma$. Thus one may equip the set of non-trivial trajectories attracted by 0 with the topology induced from $\Gamma$.

By [18], the Čech-Alexander cohomology groups $\check{H}^{*}(\Gamma)$ are isomorphic to the cohomology groups $H^{*}\left(F_{y}\right)$ of the real Milnor fibre $F_{y}=\left\{x \in f^{-1}(-y)\right.$ $|x| \leq d\}$, where $0<y \ll d \ll 1$. A more general version concerning analytic functions on manifold is presented in [19].

By [8], if $n=3$ and $f$ is harmonic then $\Gamma$ may be stratified.

Kurdyka et al. [11], in the course of proving Thom's conjecture, showed in particular that to each trajectory attracted by 0 (and so to each point in $\Gamma$ ) one may associate an element of a finite subset $L^{\prime} \subset \mathbb{Q}^{+} \times \mathbb{R}_{-}$. This way we obtain a natural partition

$$
\Gamma=\bigcup_{(l, a) \in L^{\prime}} \Gamma(l, a) .
$$

2000 Mathematics Subject Classification: Primary 37B35, 58K05; Secondary 14B05, $34 \mathrm{C} 08$.

Key words and phrases: gradient, characteristic exponents, asymptotic critical values. 


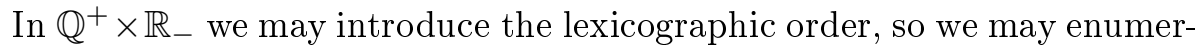
ate the elements of $L^{\prime}$ according to this order: $L^{\prime}=\left\{\left(l_{1}, a_{1}\right), \ldots,\left(l_{j}, a_{j}\right), \ldots\right.$, $\left.\left(l_{s}, a_{s}\right)\right\}$.

We will show that

$$
\Gamma\left(l_{1}, a_{1}\right) \subset \cdots \subset \bigcup_{j=1}^{i} \Gamma\left(l_{j}, a_{j}\right) \subset \cdots \subset \bigcup_{j=1}^{s} \Gamma\left(l_{j}, a_{j}\right)=\Gamma
$$

is a filtration of $\Gamma$ by closed sets, and that there are regular values $0<z_{1}<$ $\cdots<z_{i}<\cdots<z_{s}$ of the distance function $|x|$ restricted to the Milnor fibre $F_{y}$ such that each inclusion

$$
\bigcup_{j=1}^{i} \Gamma\left(l_{j}, a_{j}\right) \hookrightarrow\left\{x \in F_{y}|| x \mid \leq z_{i}\right\}
$$

induces isomorphism of Čech-Alexander cohomology groups. Hence one may apply techniques of differential topology to investigate the topology of the partition $\left\{\Gamma\left(l_{i}, a_{i}\right)\right\}$ of the set of trajectories attracted by the origin.

Among the references we list several papers $[2-7,9,10,12,13,15,17$, 20-22] devoted to geometric and topological properties of solutions of the gradient equation.

2. Preliminaries. Let $f: \mathbb{R}^{n}, 0 \rightarrow \mathbb{R}, 0$ be an analytic function defined in a neighbourhood of the origin, having a critical point at 0 . We consider the gradient $\nabla f$ of $f$. We will denote by $x(t)$ a trajectory of this vector field, that is, a curve satisfying

$$
\dot{x}(t)=\nabla f(x(t)) .
$$

It is easy to see that $\frac{d}{d t} f(x(t))>0$ unless $x(t)$ is constant, that is, $f$ is increasing along the trajectory $x(t)$. For $x$ with $f(x) \leq 0$ and sufficiently close to the origin, we denote by $\tau_{x}$ the set of points on the trajectory passing through $x$ belonging to $\{y \mid f(y) \geq f(x)\}$. Denote by $\omega(x) \in f^{-1}(0)$ either the intersection point of $\tau_{x}$ and $f^{-1}(0)$ or the limit point of the trajectory if it tends to $f^{-1}(0)$. It is well known that $\omega$ is a strong deformation retraction. that

There is a neighbourhood $U_{0}$ of the origin, $0<\varrho<1$ and $c_{\varrho}, c_{f}>0$ such

$$
\begin{gathered}
|\nabla f(x)| \geq c_{\varrho}|f(x)|^{\varrho}, \\
|x||\nabla f(x)| \geq c_{f}|f(x)|,
\end{gathered}
$$

for $x \in U_{0}$. Inequality (2.1) is due to Łojasiewicz (see [14]), and (2.2) is known as the Bochnak--Lojasiewicz inequality (see [1]). In particular as a consequence of $(2.1)$ we have $\nabla f^{-1}(0) \subseteq f^{-1}(0)$. 
The gradient $\nabla f(x)$ splits into its radial component $\frac{\partial f}{\partial r}(x) \frac{x}{|x|}$ and the spherical one $\nabla^{\prime} f(x)=\nabla f(x)-\frac{\partial f}{\partial r}(x) \frac{x}{|x|}$. We shall denote $x /|x|$ by $\partial / \partial r$ and $\partial f / \partial r$ by $\partial_{r} f$. We will also often write $r$ instead of $|x|$. Then

$$
\nabla f=\nabla^{\prime} f+\partial_{r} f \frac{\partial}{\partial r}
$$

and

$$
|\nabla f|^{2}=\left|\nabla^{\prime} f\right|^{2}+\left|\partial_{r} f\right|^{2}
$$

Now let $y, d$ be such that $0<y \ll d \ll 1$, and $-y \in \mathbb{R}$ is a regular value of $f$. We call the set $F_{y}=\{x|| x \mid \leq d, f(x)=-y\}$ the real Milnor fibre of $f$. It is either an $(n-1)$-dimensional compact manifold with boundary or an empty set (see [16]). If $f(x) \leq-y$ and $0 \in \bar{\tau}_{x}$ then $\tau_{x} \cap f^{-1}(-y) \neq \emptyset$, because the function is increasing along the trajectory. The intersection is transversal and consists exactly of one point. This justifies

Definition. $\Gamma=\left\{x \in F_{y} \mid 0 \in \bar{\tau}_{x}\right\}=\left\{x \in F_{y} \mid \omega(x)=0\right\}$.

Nowel and the second-named author showed that each trajectory attracted by the origin intersects $F_{y}$ at a point in $\Gamma$ and the topology of the set $\Gamma$ is related to the topology of the Milnor fibre. We have (see [18])

TheOREM 1. The inclusion $\Gamma \hookrightarrow F_{y}$ induces an isomorphism

$$
\check{H}^{*}(\Gamma) \simeq H^{*}\left(F_{y}\right)
$$

where $\check{H}^{*}$ denotes the $\check{C}$ ech-Alexander cohomology groups.

3. Invariants associated with trajectories. In order to say more about the topology of the set $\Gamma$, we need some notions introduced in [11]. For $\varepsilon>0$ define

$$
W^{\varepsilon}=\left\{x|f(x) \neq 0, \varepsilon| \nabla^{\prime} f|\leq| \partial_{r} f \mid\right\} .
$$

Kurdyka et al. have defined the characteristic exponents, which are characterised by the following proposition ([11, Proposition 4.2]).

Proposition 2. There exists a finite subset of positive rationals $L \subset \mathbb{Q}^{+}$ such that for any sequence $W^{\varepsilon} \ni x \rightarrow 0$ there is a subsequence $W^{\varepsilon} \ni x^{\prime} \rightarrow 0$ and $l \in L$ such that

$$
\frac{\left|x^{\prime}\right| \partial_{r} f\left(x^{\prime}\right)}{f\left(x^{\prime}\right)} \rightarrow l .
$$

In particular, as a germ at the origin, each $W^{\varepsilon}$ is the disjoint union

$$
W^{\varepsilon}=\bigcup_{l \in L} W_{l}^{\varepsilon}
$$

where

$$
W_{l}^{\varepsilon}=\left\{x \in W^{\varepsilon}|| \frac{|x| \partial_{r} f}{f}-\left.l|\leq| x\right|^{\delta}\right\},
$$


for $\delta>0$ sufficiently small. Moreover, there exist constants $0<c_{\varepsilon}<C_{\varepsilon}$, which depend on $\varepsilon$, such that

$$
c_{\varepsilon} \leq \frac{|f|}{|x|^{l}} \leq C_{\varepsilon} \quad \text { on } W_{l}^{\varepsilon} .
$$

Fix $l>0$, not necessarily in $L$, and consider $F=f /|x|^{l}$ defined in the complement of the origin. We say that $a \in \mathbb{R}$ is an asymptotic critical value of $F$ at the origin if there exists a sequence $x \rightarrow 0, x \neq 0$, such that

$$
\begin{gathered}
|x||\nabla F(x)| \rightarrow 0, \\
F(x) \rightarrow a .
\end{gathered}
$$

By [11, Propositions 5.1 and 5.4] we have

Proposition 3. The set of asymptotic critical values of $F=f /|x|^{l}$ is finite. The real number $a \neq 0$ is an asymptotic critical value if and only if there exists a sequence $x \rightarrow 0, x \neq 0$, such that

$$
\begin{gathered}
\frac{\left|\nabla^{\prime} f(x)\right|}{\left|\partial_{r} f(x)\right|} \rightarrow 0, \\
F(x) \rightarrow a .
\end{gathered}
$$

By the above proposition, the set

$$
L^{\prime}=\left\{(l, a) \mid l \in L, a<0 \text { is an asymptotic critical value of } f /|x|^{l}\right\}
$$

is a finite subset of $\mathbb{Q}^{+} \times \mathbb{R}_{-}$. For a given characteristic exponent $l \in L$ there can be more than one asymptotic critical value $a$. By Section 6 of [11] we have

TheOREM 4. For every trajectory $x(t) \rightarrow 0$ of the gradient vector field there exists a unique pair $(l, a) \in L^{\prime}$ such that $\frac{f}{r^{l}}(x(t)) \rightarrow a$.

\section{Partition of the set of trajectories}

Definition. There is a natural partition of $\Gamma$ associated with $L^{\prime}$. Namely for $(l, a) \in L^{\prime}$,

$$
\Gamma(l, a)=\left\{\left.x \in \Gamma|f(x(t)) /| x(t)\right|^{l} \rightarrow a \text { on the trajectory } \tau_{x}\right\} .
$$

Definition. In the set $\mathbb{Q}^{+} \times \mathbb{R}_{-}$we may introduce the lexicographic order

$$
(l, a) \leq\left(l^{\prime}, a^{\prime}\right) \quad \text { if } l<l^{\prime} \text {, or } l=l^{\prime} \text { and } a \leq a^{\prime} .
$$

It is obvious that $(l, a) \leq\left(l^{\prime}, a^{\prime}\right)$ if and only if $a|x|^{l} \leq a^{\prime}|x|^{l^{\prime}}$ near the origin. We enumerate the elements of $L^{\prime}$ according to this order.

Let $\langle\cdot, \cdot\rangle$ denote the standard inner product in $\mathbb{R}^{n}$. We have the following

Lemma 5. If $(l, a) \in\left(\mathbb{Q}^{+} \times \mathbb{R}_{-}\right) \backslash L^{\prime}$ then

$$
\left\langle\nabla\left(f-a|x|^{l}\right)(x), \nabla f(x)\right\rangle>0
$$

for $x \in\left(f-a|x|^{l}\right)^{-1}(0) \backslash\{0\}$ near 0 . 
Proof. Suppose, contrary to our claim, that there is a sequence $x \rightarrow 0$, $x \neq 0$, such that $f(x)-a|x|^{l}=0$ and

$$
\begin{aligned}
0 & \geq\left\langle\nabla\left(f-a|x|^{l}\right), \nabla f\right\rangle \\
& =|\nabla f|^{2}-\left\langle l a|x|^{l-1} \frac{\partial}{\partial r}, \nabla^{\prime} f+\partial_{r} f \frac{\partial}{\partial r}\right\rangle \\
& =|\nabla f|^{2}-l a r^{l-1} \partial_{r} f=|\nabla f|^{2}-\frac{l f}{r} \partial_{r} f .
\end{aligned}
$$

Using (2.2) we have

$$
l|f|\left|\partial_{r} f\right| \geq r|\nabla f|^{2} \geq c_{f}|f||\nabla f| .
$$

Hence

$$
\frac{c_{f}}{l}|\nabla f| \leq\left|\partial_{r} f\right|
$$

which means that $x \in W^{c_{f} / l}$. By Proposition 2, there are $l^{\prime} \in L$ and a subsequence $x^{\prime}$ such that

$$
\frac{\left|x^{\prime}\right| \partial_{r} f}{f} \rightarrow l^{\prime}
$$

All $x^{\prime}$ lie in $W_{l^{\prime}}^{c_{f} / l}$, hence

$$
c \leq \frac{f}{\left|x^{\prime}\right|^{l^{\prime}}} \leq C,
$$

where $c=c_{c_{f} / l}$ and $C=C_{c_{f} / l}$. Since $f\left(x^{\prime}\right)=a\left|x^{\prime}\right|^{l}, l=l^{\prime}$ is a characteristic exponent.

We shall now prove that $a$ is an asymptotic critical value. Let us transform the inequality (4.3):

$$
0 \geq\left|\nabla^{\prime} f\right|^{2}+\left|\partial_{r} f\right|^{2}-\frac{l f}{r} \frac{\left|\partial_{r} f\right|^{2}}{\partial_{r} f}=\left|\nabla^{\prime} f\right|^{2}+\left|\partial_{r} f\right|^{2}\left(1-\frac{l f}{r \partial_{r} f}\right) .
$$

Hence

$$
\frac{\left|\nabla^{\prime} f\right|^{2}}{\left|\partial_{r} f\right|^{2}} \leq\left|1-\frac{l f}{r \partial_{r} f}\right|
$$

Since

$$
\frac{r \partial_{r} f}{f}=\frac{\left|x^{\prime}\right| \partial_{r} f\left(x^{\prime}\right)}{f\left(x^{\prime}\right)} \rightarrow l^{\prime}=l
$$

the right-hand side of the inequality (4.5) tends to 0 . So does the left-hand side and we have

$$
\frac{\left|\nabla^{\prime} f\right|}{\left|\partial_{r} f\right|}\left(x^{\prime}\right) \rightarrow 0 \quad \text { and } \quad \frac{f\left(x^{\prime}\right)}{\left|x^{\prime}\right|^{l}}=a .
$$

By Proposition 3, $a$ is an asymptotic critical value of $f / r^{l}$. 
Take $(l, a) \in \mathbb{Q}^{+} \times \mathbb{R}_{-} \backslash L^{\prime}$ and $y>0$ close to 0 such that $-y$ is a regular value of $f$. Define

$$
\Theta(l, a)=F_{y} \cap\left\{f-a|x|^{l} \leq 0\right\}=F_{y} \cap\left\{|x| \leq(y /(-a))^{1 / l}\right\} .
$$

We will show a relation between the cohomologies of $\Theta(l, a)$ and

$$
\widetilde{\Gamma}(l, a)=\bigcup_{\left(l_{i}, a_{i}\right)<(l, a)} \Gamma\left(l_{i}, a_{i}\right), \quad \text { where }\left(l_{i}, a_{i}\right) \in L^{\prime} .
$$

Theorem 6. For every $(l, a) \in \mathbb{Q}^{+} \times \mathbb{R}_{-} \backslash L^{\prime}$ and every $y>0$ small enough, $\widetilde{\Gamma}(l, a)$ is closed, and there is an inclusion

$$
\widetilde{\Gamma}(l, a) \hookrightarrow \Theta(l, a),
$$

which induces an isomorphism

$$
\check{H}^{*}(\widetilde{\Gamma}(l, a)) \cong H^{*}(\Theta(l, a)) .
$$

LEMMA 7. For every $\varepsilon>0$ there exists $\eta=\eta(\varepsilon)>0$ such that if $|x|<\eta$ then for every point $y$ on $\tau_{x}$ between $x$ and $\omega(x)$ we have $|y|<\varepsilon$.

Proof. For $a \in \tau_{x}$ denote by $\ell(x, a)$ the length of the trajectory between $x$ and $a$. From the Eojasiewicz inequality (2.1) it follows (see [11]) that for $x$ close to the origin

$$
\ell(x, a) \leq c_{\varrho}(1-\varrho)^{-1}\left[|f(x)|^{1-\varrho}-|f(a)|^{1-\varrho}\right] .
$$

As $a \rightarrow \omega(x)$ we get

$$
\ell(x, \omega(x)) \leq c_{\varrho}(1-\varrho)^{-1}|f(x)|^{1-\varrho}=c_{1}|f(x)|^{1-\varrho} .
$$

By continuity of $f$ there exists $\eta, 0<\eta<\varepsilon / 2$, such that for $|x|<\eta$,

$$
\ell(x, \omega(x)) \leq c_{1}|f(x)|^{1-\varrho}<\varepsilon / 2 .
$$

That is, for $x^{\prime}$ between $x$ and $\omega(x)$,

$$
\left|x^{\prime}\right| \leq|x|+\ell\left(x, x^{\prime}\right)<\frac{\varepsilon}{2}+\frac{\varepsilon}{2}=\varepsilon .
$$

Define $A_{\leq}=\left\{\left.x|-y \leq f(x) \leq a| x\right|^{l}\right\}$ and $A_{=}=\left\{\left.x|-y \leq f(x)=a| x\right|^{l}\right\}$. If $y$ is small enough then $A_{\leq}$is bounded by $A_{=}$and $\Theta(l, a)$. By Corollary 6 , $A_{=}$and $\Theta(l, a)$ intersect transversally.

If $x \in \Theta(l, a)$ then $\nabla f(x)$ is normal to $\Theta(l, a)$ and points into $A_{\leq}$. If $x \in A_{=} \backslash\{0\}$ then $\nabla\left(f-a|x|^{l}\right)$ is normal to $A_{=}$and points away from $A_{\leq}$.

We consider a mapping $\gamma: \Theta(l, a) \rightarrow A_{=}$such that $\gamma(x)$ is the point of intersection of the trajectory $\tau_{x}$ with the set $A_{=}$or $\gamma(x)=\omega(x)=0$ if $\tau_{x}$ does not intersect $A_{=}$.

Lemma 8. $\gamma$ is well defined, and $\gamma^{-1}(0)=\widetilde{\Gamma}(l, a)$.

Proof. Consider trajectories starting from $\Theta(l, a)$. Some of them will stay in the set $A_{\leq}$and others will leave it forever. (A trajectory cannot get back to $A_{\leq}$, because for a point $x \in A_{=} \backslash\{0\}$ we have $\left\langle\nabla\left(f-a|x|^{l}\right)(x), \nabla f(x)\right\rangle>0$. 
The angle between the gradients $\nabla\left(f-a|x|^{l}\right)(x)$ and $\nabla f(x)$ is less than $\pi / 2$, so the trajectory passing through $x$ leaves $A_{\leq}$.)

Consider a trajectory $\tau_{x}$ which stays in $A_{\leq}$. By the Eojasiewicz inequality (2.1), $\nabla f$ does not vanish on $A_{\leq} \backslash\{0\}$. Hence $x(t) \rightarrow 0$, i.e. $\gamma(x)=\omega(x)=0$ and $x \in \Gamma$. That is, we proved $\gamma$ is well defined. By Theorem 4 there is $\left(l_{i}, a_{i}\right) \in L^{\prime}$ such that $f(x(t)) /|x(t)|^{l_{i}} \rightarrow a_{i}$.

The trajectory stays inside $A_{\leq}$, so

$$
f(x(t))-a|x(t)|^{l} \leq 0 .
$$

For every $\varepsilon>0$, if $x(t)$ is sufficiently close to the origin we have

$$
\left(a_{i}-\varepsilon\right)|x(t)|^{l_{i}}<f(x(t)) \leq a|x(t)|^{l} .
$$

Therefore $l_{i}<l$ or $l_{i}=l$ and $a_{i}-\varepsilon<a$ for every $\varepsilon>0$. Hence

$$
\left(l_{i}, a_{i}\right) \leq(l, a) .
$$

Since $(l, a) \notin L^{\prime},\left(l_{i}, a_{i}\right)<(l, a)$.

Now consider a trajectory $\tau_{x}$ which leaves $A_{\leq}$, i.e. $\gamma(x) \neq 0$. Then for $t$ large enough we have $f(x(t))>a|x(t)|^{l}$. If $\tau_{x}$ starts from $\Gamma$, then $x(t) \rightarrow 0$ and there is $\left(l_{i}, a_{i}\right) \in L^{\prime}$ such that $f(x(t)) /|x(t)|^{l_{i}} \rightarrow a_{i}$. For every $\varepsilon>0$,

$$
\left(a_{i}+\varepsilon\right)|x(t)|^{l_{i}}>f(x(t))>a|x(t)|^{l}
$$

if $x(t)$ is sufficiently close to the origin. Applying similar arguments to the above we have $\left(l_{i}, a_{i}\right)>(l, a)$. Similarly for a trajectory which starts from $\Gamma$ outside $\Theta(l, a)$ : it cannot enter the set $A_{\leq}$and hence $\left(l_{i}, a_{i}\right)$ corresponding to that trajectory is greater than $(l, a)$.

LEMMA 9. $\gamma$ is continuous, and $\gamma$ restricted to $\Theta(l, a) \backslash \widetilde{\Gamma}(l, a)$ is a homeomorphism onto $\operatorname{Im} \gamma \backslash\{0\}=A_{=} \backslash\{0\}$. In particular, $\widetilde{\Gamma}(l, a)$ is compact.

Proof. Consider $x \in \Theta(l, a)$ such that $\gamma(x) \neq 0$. Then $\tau_{x}$ is transversal to $\Theta(l, a)$ at $x$ and to $A_{=}$at $\gamma(x)$, therefore $\gamma$ is a Poincaré mapping in some neighbourhood of $x$. Hence $\gamma$ is a local homeomorphism at $x$.

Now take $x$ such that $\gamma(x)=0$. Then $\tau_{x} \subset A_{\leq}$and $0 \in \bar{\tau}_{x}$. Fix an $\varepsilon>0$. There is $x^{\prime} \in \tau_{x}$ such that $\left|x^{\prime}\right|<\eta / 2$, where $\eta=\bar{\eta}(\varepsilon)$ comes from Lemma 7 . Now consider a neighbourhood $V$ of $x^{\prime}$ of diameter $\eta / 2$ contained in $A_{\leq}$. Reversing trajectories we get an open neighbourhood $W \subset \Theta(l, a)$ of $x$ such that $|\gamma(y)|<\varepsilon$ for $y \in W$.

Lemma 10. For every open neighbourhood $U$ of $\widetilde{\Gamma}(l, a)$ in $\Theta(l, a), \gamma(U)$ is an open neighbourhood of 0 in $\operatorname{Im} \gamma=A_{=}$.

Proof. Rewrite the proof of Lemma 9 in [18] substituting $\Theta(l, a)$ for $F_{r}$ and $\operatorname{Im} \gamma$ for $Z_{r}$.

Proof of Theorem 6. The inclusion $\widetilde{\Gamma}(l, a) \subseteq \Theta(l, a)$ follows from the fact that $\widetilde{\Gamma}(l, a)=\gamma^{-1}(0)$ as stated in Lemma 8 . 
In order to prove that the inclusion induces an isomorphism of ČechAlexander cohomology groups, we will construct a descending family $\Theta(l, a)$ $=U_{1} \supset U_{2} \supset \cdots$ of open neighbourhoods of $\widetilde{\Gamma}(l, a)$ in $\Theta(l, a)$, which satisfies

(u1) every inclusion $U_{n+1} \subset U_{n}$ is a homotopy equivalence,

(u2) for every neighbourhood $U$ of $\widetilde{\Gamma}(l, a)$ in $\Theta(l, a)$ there is $n$ such that $U_{n} \subset U$.

The set $\operatorname{Im} \gamma=A_{=}=\left\{\left.x|f=a| x\right|^{l},|x| \leq(y /(-a))^{1 / l}\right\}$, for $y$ small enough, is homeomorphic to a cone with vertex at 0 , so there is a descending family $A_{=}=V_{1} \supset V_{2} \supset \cdots$ of open neighbourhoods of 0 in $A_{=}$such that every inclusion is a homotopy equivalence and for every open neighbourhood $V$ of 0 in $A_{=}$there is $n$ such that $V_{n} \subset V$. We put $U_{n}=\gamma^{-1}\left(V_{n}\right)$. Clearly $\left\{U_{n}\right\}$ is a family of open neighbourhoods of $\widetilde{\Gamma}(l, a)$ in $\Theta(l, a)$. The mapping $\gamma$ restricted to $\Theta(l, a) \backslash \widetilde{\Gamma}(l, a)$ is a homeomorphism onto $A=\backslash\{0\}$, hence (u1) holds. If $U$ is an open neighbourhood of $\widetilde{\Gamma}(l, a)$ then by Lemma 10, $\gamma(U)$ is an open neighbourhood of 0 . There is $n$ such that $V_{n} \subset \gamma(U)$; then $U_{n} \subset U$, so (u2) holds.

As the family $\left\{U_{n}\right\}$ is cofinal in the family of all open neighbourhoods of $\widetilde{\Gamma}(l, a)$ in $\Theta(l, a)$ ordered by $\supseteq$, we have an isomorphism of direct limits

$$
\underset{U}{\lim _{U}} H^{*}(U) \cong \underset{U_{n}}{\longrightarrow} H^{*}\left(U_{n}\right)=\check{H}^{*}(\widetilde{\Gamma}(l, a)) .
$$

Since $H^{*}\left(U_{n}\right) \cong H^{*}(\Theta(l, a))$ by $(\mathrm{u} 1)$, the theorem holds.

For given $l \in \mathbb{Q}^{+}$and $y,(y /(-a))^{1 / l}$ is a regular value of $|x|_{\mid F_{y}}$, for almost all $a \in \mathbb{R}_{-}$. In that case $\Theta(l, a)$ is either void or a compact $(n-1)$-manifold with boundary.

Proposition 11. For each $(l, a) \in\left(\mathbb{Q}^{+} \times \mathbb{R}_{-}\right) \backslash L^{\prime}$ and each $y>0$ small enough, $z=(y /(-a))^{1 / l}$ is a regular value for $|x|_{\mid F_{y}}$ and the inclusion

$$
\widetilde{\Gamma}(l, a)=\bigcup_{\left(l_{i}, a_{i}\right)<(l, a)} \Gamma\left(l_{i}, a_{i}\right) \hookrightarrow F_{y} \cap\{|x| \leq z\}
$$

induces an isomorphism of $\check{C}$ ech-Alexander cohomology groups.

Proof. Consider the set of critical values of $|x|_{\mid F_{y}}$. For a given $y$ we have finitely many critical values $w_{1}(y), \ldots, w_{p}(y)$. We can treat $w_{j}(y)$ as a real function. The graph of $w_{j}$ is a subanalytic set. Since it lies in the plane, it is semianalytic. Hence we can write the Puiseux expansion for each $w_{j}$ (see [14]):

$$
w_{j}(y)=b y^{m}+\cdots \quad\left(b>0, m \in \mathbb{Q}_{+}\right) .
$$

We will show that $\left(1 / m,-b^{-1 / m}\right) \in L^{\prime}$.

By the curve selection lemma we can choose a curve $\xi(r)$ of critical points corresponding to $w_{j}$. We parametrize the curve by the distance to the origin. 
Put $y(r)=-f(\xi(r))$. That is, $\xi(r) \in F_{y(r)}$ is a critical point of $|x|_{\mid F(y(r))}$ such that

$$
r=|\xi(r)|=w_{j}(y(r))=b(y(r))^{m}+\cdots .
$$

We can also write a Puiseux expansion of $f$ along this curve,

$$
f(\xi(r))=-\alpha r^{q}+\cdots \quad\left(\alpha>0, q \in \mathbb{Q}_{+}\right) .
$$

Thus

$$
y(r)=\alpha r^{q}+\cdots
$$

By (4.7) and (4.6) we get

$$
r=b\left(\alpha r^{q}\right)^{m}+\cdots=b \alpha^{m} r^{q m}+\cdots
$$

along the curve $\xi(r)$. Hence $q m=1$ and $b \alpha^{m}=1$. That is,

$$
f(\xi(r))=-b^{-1 / m} r^{1 / m}+\cdots .
$$

The curve $\xi(r)$ consists of critical points of $|x|_{\mid F_{y(r)}}$ and therefore on $\xi(r)$ we have $\left|\nabla^{\prime} f\right| \equiv 0,|\nabla f|=\left|\partial_{r} f\right|$. For every $\varepsilon>0$ we have $\varepsilon\left|\nabla^{\prime} f\right|<\left|\partial_{r} f\right|$, and that means the curve $\xi$ lies in every $W^{\varepsilon}$, so there exists a characteristic exponent $l^{\prime}$ such that $\xi$ lies in $W_{l^{\prime}}^{\varepsilon}$.

Since $f(\xi(r)) /|\xi(r)|^{1 / m} \rightarrow-b^{-1 / m}$, it follows that $l^{\prime}=1 / m \in L$ by the last statement of Proposition 2. By Proposition $3,-b^{-1 / m}$ is the corresponding asymptotic critical value for $f / r^{1 / m}$. In particular, $\left(1 / m,-b^{-1 / m}\right) \in L^{\prime}$. Assume that $(l, a) \notin L^{\prime}$. If $y$ is small enough, then $(y /(-a))^{1 / l}=(-a)^{-1 / l} y^{1 / l}$ is different from any $w_{j}(y)$. Hence it is a regular value for $|x|_{\mid F_{y}}$.

Now it is enough to apply Theorem 7 .

The proof above gives us even more:

THEOREM 12. Let $f: \mathbb{R}^{n}, 0 \rightarrow \mathbb{R}, 0$ be an analytic function defined in a neighbourhood of the origin, having a critical point at 0 . For each y small enough there is a finite sequence $0<z_{1}<\cdots<z_{i}<\cdots<z_{s}$ of regular values of $|x|_{\mid F_{y}}$ such that

$$
\Gamma\left(l_{1}, a_{1}\right) \subset \cdots \subset \bigcup_{j=1}^{i} \Gamma\left(l_{j}, a_{j}\right) \subset \cdots \subset \bigcup_{j=1}^{s} \Gamma\left(l_{j}, a_{j}\right)=\Gamma
$$

is a filtration of $\Gamma$ by closed sets, and the inclusions

$$
\bigcup_{j=1}^{i} \Gamma\left(l_{j}, a_{j}\right) \hookrightarrow\left\{x \in F_{y}|| x \mid \leq z_{i}\right\}
$$

induce isomorphisms of $\check{C}$ ech-Alexander cohomology groups. One can take $z_{i}=(y /(-a))^{1 / l}$, where $\left(l_{i}, a_{i}\right)<(l, a)<\left(l_{i+1}, a_{i+1}\right)$.

Proof. Let $s$ be the cardinality of $L^{\prime}$. As we have seen in the proof of Corollary 11 , if $(l, a) \notin L^{\prime}$ then $(y /(-a))^{1 / l}$ is a regular value of $|x|_{\mid F_{y}}$. Since 
$L^{\prime}$ is totally ordered by the lexicographic ordering, for every $i$ we can choose a pair $(l, a)$ such that

$$
\left(l_{i}, a_{i}\right)<(l, a)<\left(l_{i+1}, a_{i+1}\right),
$$

where $\left(l_{s+1}, a_{s+1}\right)$ is greater than any pair in $L^{\prime}$. Set $z_{i}(y)=(y /(-a))^{1 / l}$. One can easily see that $z_{i}<z_{i+1}$ and $z_{i}(y) \neq w_{j}(y)$ for sufficiently small $y$.

By Proposition 11, the vertical inclusions induce isomorphisms of the Čech-Alexander cohomology groups.

The above theorem shows that applying well known methods of differential topology and Morse theory to the distance function $|x|$ on the Milnor fibre may provide important information about the topology of families of trajectories of an analytic gradient vector field with given characteristic exponent and asymptotic critical value.

Acknowledgments. The authors wish to express their gratitude to the referee for helpful comments.

Research partially supported by grant BW 5100-5-0148-4, and the European Community IHP-Network RAAG (HPRN-CT-2001-00271).

\section{References}

[1] J. Bochnak and S. Łojasiewicz, A converse to the Kuiper-Kuo theorem, in: Proc. Liverpool Singularities Symposium I, Lecture Notes in Math. 192, Springer, New York, 1971, 254-261.

[2] F. Cano, R. Moussu et F. Sanz, Oscillation, spiralement, tourbillonnement, Comment. Math. Helv. 75 (2000), 284-318.

[3] O. Cornea, Homotopical dynamics III: Real singularities and Hamiltonian flows, Duke Math. J. 209 (2001), 183-204.

[4] D. D'Acunto and V. Grandjean, On gradient at infinity of real polynomials, preprint.

[5] N. Dancer, Degenerate critical points, homotopy indices and Morse inequalities, J. Reine Angew. Math. 382 (1984), 1-22.

[6] P. Fortuny and F. Sanz, Gradient vector fields do not generate twister dynamics, J. Differential Equations 174 (2001), 91-100.

[7] V. Grandjean, On the limit set at infinity of gradient of semialgebraic function, preprint.

[8] P. Goldstein, Flows of gradients of harmonic functions on $\mathbb{R}^{3}, \mathrm{PhD}$. Thesis, Warsaw Univ., 2004 (in Polish).

[9] F. Ichikawa, Thom's conjecture on singularities of gradient vector fields, Kodai Math. J. 15 (1992), 134-140.

[10] K. Kurdyka, On the gradient conjecture of R. Thom, in: Seminari di Geometria 19981999, Università di Bologna, Istituto di Geometria, Dipartamento di Matematica, $2000,143-151$.

[11] K. Kurdyka, T. Mostowski and A. Parusiński, Proof of the gradient conjecture of R. Thom, Ann. of Math. 152 (2000), 763-792.

[12] K. Kurdyka and A. Parusiński, $w_{f}$-stratification of subanalytic functions and the Eojasiewicz inequality, C. R. Acad. Sci. Paris Sér. I 318 (1994), 129-133. 
[13] J.-M. Lion, R. Moussu et F. Sanz, Champs de vecteurs analytiques et champs de gradients, Ergodic Theory Dynam. Systems 22 (2002), 525-534.

[14] S. Łojasiewicz, Ensembles semi-analytiques, IHES, 1965.

[15] - Sur les trajectoires du gradient d'une fonction analytique, in: Seminari di Geometria 1982-1983, Università di Bologna, Istituto di Geometria, Dipartamento di Matematica, 1984, 115-117.

[16] J. Milnor, Singular Points on Complex Hypersurfaces, Ann. of Math. Stud. 61, Princeton Univ. Press, Princeton, NJ, 1968.

[17] R. Moussu, Sur la dynamique des gradients. Existence de variétés invariants, Math. Ann. 307 (1997), 445-460.

[18] A. Nowel and Z. Szafraniec, On trajectories of analytic gradient vector fields, J. Differential Equations 184 (2002), 215-223.

[19] - - -, On trajectories of analytic gradient vector fields on analytic manifolds, Topol. Methods Nonlinear Anal., to appear.

[20] F. Sanz, Non-oscillating solutions of analytic gradient vector fields, Ann. Inst. Fourier (Grenoble) 48 (1998), 1045-1067.

[21] F. Takens, The minimal number of critical points of a function on a compact manifold and the Lusternik-Schnirelmann category, Invent. Math. 6 (1968), 197-244.

[22] R. Thom, Problèmes rencontrés dans mon parcours mathématique: un bilan, Publ. Math. IHES 70 (1989), 200-214.

Institute of Mathematics

University of Gdańsk

Wita Stwosza 57

80-952 Gdańsk, Poland

E-mail: adam.dzedzej@math.univ.gda.pl

zbigniew.szafraniec@math.univ.gda.pl

Reçu par la Rédaction le 24.9.2004

Révisé le 24.5.2005 